Pacific Journal of Mathematics

ON GINSBURG-ISBELL DERIVATIVES AND RANKS O 


\title{
ON GINSBURG-ISBELL DERIVATIVES AND RANKS OF METRIC SPACES
}

\section{AARNO HOHTI}

\begin{abstract}
The paper deals with the following problem: given a metric space, is there a countable ordinal $\alpha$ such that the $\alpha$ th successive Ginsburg-Isbell derivative of the metric uniformity contains every open cover of the space? In addition to other results we show that a separable metric space has the above property if and only if it is complete and $\sigma$-compact.
\end{abstract}

1. Introduction. In their fundamental 1959 paper [1], Ginsburg and Isbell defined, for a filter $\mu$ of coverings, the successive derivatives $\mu^{(\alpha)}$ as follows. Let $\mu^{(0)}=\mu$. If $\mu^{(\alpha)}$ is defined for all $\alpha<\beta$ and $\beta$ is a limit ordinal, let $\mu^{(\beta)}=\bigcup\left\{\mu^{(\alpha)}: \alpha<\beta\right\}$; if $\beta=\alpha+1$, let $\mu^{(\beta)}$ consist of all covers $\left\{V_{l} \cap W_{j}^{i}\right\}_{l, j}$, where $\left\{V_{i}\right\}_{i} \in \mu^{(\alpha)}$ and, for each $i,\left\{W_{j}^{i}\right\} \in \mu^{(\alpha)}$. There is the least derivative $\mu^{(\alpha)}$ for which $\mu^{(\alpha+1)}=\mu^{(\alpha)}$; denote that derivative by $\lambda \mu$. It was shown in [1] that for any complete metric space $\rho X, \lambda \rho$ is the fine uniformity of $X$. On the other hand, we proved in [2] that if every binary open cover of $X$ belongs to $\lambda \rho$, then $\rho X$ is complete. Thus, in a sense, in the class of metric spaces the complete spaces form the subclass of objects in which every open cover can be approximated by "piecewise uniform" covers. Here we shall consider the class of spaces for which the approximation can be obtained by taking countably many successive derivatives. To facilitate the discussion a new derivative $\mu_{0}^{(\alpha)}$ (slower than the original one) is introduced in $§ 2$.

J. Nagata [5] called a uniform space $\mu X \mu$-normal if every two disjoint closed subsets of $X$ can be separated by $\mu$-uniform neighbourhoods. It can be shown that $\mu X$ is $\mu$-normal if and only if every finite cover of $X$ is $\mu$-uniform. Nagata proved that a metric space $\rho X$ is $\mu$-normal if and only if there is a compact subset $H$ of $X$ such that for any $\varepsilon>0$, the complement of $S_{\rho}(H, \varepsilon)$ is uniformly discrete. In $\S 3$ we extend Nagata's result to metric spaces $\rho X$ for which there is a countable ordinal $\alpha$ such that each finite open cover of $X$ belongs to $\rho_{0}^{(\alpha)}$. Such spaces are $C$-scattered in the sense of Telgársky: a topological space $X$ is called $C$-scattered if every nonempty closed subspace of $X$ contains a point with a compact neighbourhood. (The converse of that statement will be proved for separable complete metric spaces in $\S 4$.) We conclude $\$ 3$ by proving a theorem on ranks of finite products of metric spaces. 
$\S 4$ deals with complete separable metric spaces. The main result is that our extension of Nagata's theorem yields an analysis of $\sigma$-compact complete metric spaces: if $\rho X$ is $\sigma$-compact and complete, then there is a countable ordinal $\alpha$ such that each open cover of $X$ belongs to $\rho^{(\alpha)}$. Our results in $\$ 3$ can then be applied to give an analysis of those spaces.

2. A new derivative. To prove our theorems it is helpful to introduce a new derivative slow enough for inductive purposes. Let $\mu$ be a filter of coverings ordered by the relation of refinement. Let $\mu_{0}^{(0)}=\mu$. If $\mu_{0}^{(\alpha)}$ is defined for all $\alpha<\beta$, and $\beta$ is a limit ordinal, then $\mu_{0}^{(\beta)}=\bigcup\left\{\mu_{0}^{(\alpha)}\right.$ : $\alpha<\beta\}$; on the other hand, if $\beta=\alpha+1$, then $\mu_{0}^{(\beta)}$ consists of all covers $\left\{V_{i} \cap W_{j}^{i}\right\}_{i, j}$ where $\left\{V_{i}\right\}_{i} \in \mu$ and, for each $i,\left\{W_{j}^{i}\right\}_{j} \in \mu_{0}^{(\alpha)}$. (Thus, in the notation of [6], $\mu^{(\alpha+1)}=\mu^{(\alpha)} / \mu^{(\alpha)}$, whereas $\mu_{0}^{(\alpha+1)}=\mu_{0}^{(\alpha)} / \mu$.) The restriction of a cover $\mathcal{V}$ to $A$ is denoted by $\mathscr{V} \mid A$. In a similar vein, the family $\{\mathfrak{V} \mid A: \mathcal{V} \in \mu\}$ of the restrictions of members from $\mu$ will be denoted by $\mu \mid A$. The following lemma can be proved by transfinite induction.

LEMMA 2.1. Let $\mu$ be a filter of coverings of $X$ and let $A \subset X$. If $\mathfrak{V}$ is a cover of $A$, then $\mathcal{V} \in(\mu \mid A)_{0}^{(\alpha)}$ if and only if there is a $\mathscr{W} \in \mu_{0}^{(\alpha)}$ such that था $\mid A<\mathcal{T}$.

In the following lemma we will use the Hessenberg natural sum $\oplus$ of ordinal numbers. (See [4], p. 252.) It is enough to know that $\oplus$ is commutative and strictly increasing.

LEMMA 2.2. Let $\alpha$ and $\beta$ be ordinals. If $\left\{V_{i}\right\}_{i} \in \mu_{0}^{(\alpha)}$ and, for each $i$, $\left\{V_{i} \cap W_{j}^{i}\right\}_{j} \in\left(\mu \mid V_{i}\right)_{0}^{(\beta)}$, then $\left\{V_{i} \cap W_{j}^{i}\right\}_{i, j} \in \mu_{0}^{(\alpha \oplus \beta+1)}$.

Proof. We will proceed by induction on $\alpha$. If $\alpha=0$, then the contention is true by definition and Lemma 2.1. For the induction hypothesis, suppose that it holds for $0 \leq \alpha<\gamma$. Let $\left\{V_{i}\right\} \in \mu_{0}^{(\gamma)}$. If $\gamma$ is a limit ordinal, then there is a $\nu<\gamma$ with $\left\{V_{i}\right\} \in \mu_{0}^{(\nu)}$ and the induction hypothesis implies that $\left\{V_{i} \cap W_{j}^{i}\right\}_{i, j} \in \mu_{0}^{(\nu \oplus \beta+1)} \subset \mu_{0}^{(\gamma \oplus \beta+1)}$. On the other hand, suppose $\gamma=$ $\nu+1$. Then there is a uniform cover $\left\{U_{s}\right\}_{s}$ of $X$ and for each $s$ a cover $\left\{G_{t}^{s}\right\}_{t} \in \mu_{0}^{(\nu)}$ such that $\left\{V_{i}\right\}_{i}>\left\{U_{s} \cap G_{t}^{s}\right\}_{s, t}$. For all pairs $(s, t)$ there exists a $\left\{\tilde{W}_{j}^{s t}\right\}_{j} \in \mu_{0}^{(\beta)}$ whose restriction to $U_{s} \cap G_{t}^{s}$ refines the original covers $\left\{W_{j}^{i}\right\}_{j}$. The restriction of $\left\{\tilde{W}_{j}^{s t}\right\}_{j}$ to $G_{t}^{s}$ belongs to $\left(\mu \mid G_{t}^{s}\right)_{0}^{(\beta)}$; hence by the induction hypothesis $\left\{G_{t}^{s} \cap \tilde{W}_{j}^{s t}\right\}_{t, j} \in \mu_{0}^{(\nu \oplus \beta+1)}$. Now $\nu \oplus \beta+1=(\nu+1)$ $\oplus \beta=\gamma \oplus \beta$. Since $\left\{U_{s}\right\}$ is a uniform cover of $X$, we have

$$
\left\{U_{s} \cap G_{t}^{s} \cap \tilde{W}_{j}^{s t}\right\}_{s, t, j} \in \mu_{0}^{(\gamma \oplus \beta+1)} .
$$

Therefore $\left\{V_{i} \cap W_{j}^{i}\right\}_{i, j}$ belongs to $\mu_{0}^{(\gamma \oplus \beta+1)}$. 
LEMMA 2.3. (i) $\mu_{0}^{(\alpha)} \subset \mu^{(\alpha)}$ for all $\alpha$; (ii) for each $\alpha$ there is a $\sigma(\alpha)$ such that $\mu^{(\alpha)} \subset \mu_{0}^{(\sigma(\alpha))}$. Moreover, if $\tau$ is an infinite regular ordinal, then we can choose $\sigma(\alpha)$ so that $\sigma(\alpha)<\tau$.

Proof. The first statement being obvious, we prove (ii) only. We will use induction on $\alpha$. Clearly $\mu^{(0)} \subset \mu_{0}^{(0)}$. Suppose for each $\alpha<\beta$ there is a $\sigma(\alpha)$ such that $\mu^{(\alpha)} \subset \mu_{0}^{(\sigma(\alpha))}$. Moreover, let $\tau$ be an infinite regular ordinal with $\beta<\tau$ and $\sigma(\alpha)<\tau$ for $\alpha<\beta$. If $\beta$ is a limit ordinal, then $\mu^{(\beta)}=$ $\bigcup\left\{\mu^{(\alpha)}: \alpha<\beta\right\}$. Since $\tau$ is regular, we have $\gamma=\sup \{\sigma(\alpha): \alpha<\beta\}<\tau$. It is easy to see that $\mu^{(\beta)} \subset \mu_{0}^{(\gamma)}$. Therefore, we can assume $\beta$ is a successor ordinal, say $\beta=\nu+1$. Each element of $\mu^{(\nu+1)}\left(=\mu^{(\nu)} / \mu^{(\nu)}\right)$ has the form $\mathcal{V}=\left\{V_{i} \cap W_{j}^{i}\right\}_{i, j}$, where $\left\{V_{i}\right\}_{i},\left\{W_{j}^{i}\right\}_{j} \in \mu^{(\nu)}$. By the induction hypothesis $\mu^{(\nu)} \subset \mu_{0}^{(\sigma(\nu))}$ and $\sigma(\nu)<\tau$. Note that $\left\{V_{i} \cap W_{j}^{i}\right\}_{j} \in\left(\mu \mid V_{i}\right)_{0}^{(\sigma(\nu))}$ for all $i$. It follows from Lemma 2.2 that $\left\{V_{i} \cap W_{j}^{i}\right\}_{i, j} \in \mu_{0}^{(\sigma(\nu) \oplus \sigma(\nu)+1)}$. Since $\sigma(\nu) \oplus$ $\sigma(\nu)+1<\tau$, we define $\sigma(\beta)=\sigma(\nu) \oplus \sigma(\nu)+1$.

Lemma 2.3 shows that if we want to prove a statement by induction on the Ginsburg-Isbell derivatives $\mu^{(\alpha)}$ we need only work with the new derivatives $\mu_{0}^{(\alpha)}$. In many cases these new derivatives are easier to handle.

3. A characterization of metric spaces of countable rank. Let $\mu X$ be a uniform space. The space $\mu X$ is called ranked if $\lambda \mu$ is the fine uniformity of $X$. We say that $\mu X$ is finitarily ranked if every finite cozero-set cover of $X$ belongs to $\lambda \mu$. The rank (resp. Ginsburg-Isbell rank) of $\mu X$ is the least ordinal $\alpha$ such that $\mu_{0}^{(\alpha)}$ (resp. $\mu^{(\alpha)}$ ) is the fine uniformity of $X$. The finitary rank (resp. finitary Ginsburg-Isbell rank) of $\mu X$ is the least ordinal $\alpha$ such that every finite cozero-set cover of $X$ belongs to $\mu_{0}^{(\alpha)}\left(\right.$ resp. $\left.\mu^{(\alpha)}\right)$. Thus, a metric space is finitarily ranked if and only if the space is complete. (See [2], p. 19.)

In this section we will extend Nagata's theorem ([5], Theorem 3) of $\mu$-normal spaces and our theorem ([2], Theorem 4.2.7) on $C$-normal spaces. (In fact, a metric space is $C$-normal if and only if the space is of rank $\leq 1$. For example, a uniformly locally compact space is of rank $\leq 1$.) The proofs of 3.2-3.6 are similar to those given for 4.2.4-4.2.8 in [2] modulo certain complications. For this reason we prove only Lemma 3.2 and leave the rest to the reader. However, we first establish a useful lemma.

LEMMA 3.1. Let $\mu X$ be a uniform space and let $\alpha$ be an ordinal. The following conditions are equivalent:

(i) if $E$ and $F$ are closed and disjoint subsets of $X$, then there is a $\mathcal{V} \in \mu_{0}^{(\alpha)}$ such that $\operatorname{St}(E, \mathcal{V}) \subset X \sim F$; 
(ii) every binary open cover of $X$ belongs to $\mu_{0}^{(\alpha)}$;

(iii) every finite open cover of $X$ belongs to $\mu_{0}^{(\alpha)}$.

Proof. The equivalence of (i) and (ii) is obvious. Thus, it is enough to show that (ii) implies (iii). Consider the following statement $P(\alpha)$ : if $\mathscr{V}=\left\{V_{1}, \ldots, V_{n}\right\}$ is an open cover of a uniform space $\nu Y,\left\{W_{1}, \ldots, W_{n}\right\}$ is an open refinement of $\mathfrak{V}$ with $\mathrm{cl}_{Y} W_{i} \subset V_{i}$ and each of the binary covers $\left\{V_{i}, Y \sim \mathrm{cl}_{Y} W_{i}\right\}$ belongs to $\nu_{0}^{(\alpha)}$, then $\mathcal{T} \in \nu_{0}^{(\alpha)}$. It is not difficult to see that $P(0)$ is valid. Indeed, let $\left\{V_{i}, Y \sim \mathrm{cl}_{Y} W_{i}\right\}$ be a uniform cover of $\nu Y$ for $1 \leq i \leq n$. Choose a uniform cover $\mathcal{Q}$ such that for each $i$, the cover $\mathcal{Q}$ refines $\left\{V_{i}, Y \sim \operatorname{cl}_{Y} W_{i}\right\}$. Then for each $U \in \mathcal{U}$ there is a $V_{i}$ such that $U \subset V_{i}$, because otherwise $U \subset \cap\left\{Y \sim \mathrm{cl}_{Y} W_{i}: 1 \leq i \leq n\right\}=Y-$ $\cup\left\{\operatorname{cl}_{Y} W_{i}: 1 \leq i \leq n\right\}=\varnothing$. Consequently, $\mathscr{V}$ is a uniform cover of $\nu Y$.

Now suppose $P(\alpha)$ holds whenever $\alpha<\beta$. To show that $P(\beta)$ holds, let $\mathcal{V}=\left\{V_{1}, \ldots, V_{n}\right\}, \nu Y$ and $\left\{W_{1}, \ldots, W_{n}\right\}$ be as above and assume that each of the binary covers $\mathcal{G}_{i}=\left\{V_{i}, Y \sim \mathrm{cl}_{Y} W_{i}\right\}$ belongs to $\mu_{0}^{(\beta)}$. If $\beta$ is a limit ordinal, then for each $i$ there is an $\alpha(i)<\beta$ such that $\mathcal{G}_{i} \in \mu_{0}^{(\alpha(i))}$. Then $\mathcal{G}_{1}, \ldots, \mathcal{G}_{n} \in \mu_{0}^{(\alpha)}$, where $\alpha=\max \{\alpha(1), \ldots, \alpha(n)\}<\beta$ and the induction hypothesis shows that $\mathscr{V} \in \mu_{0}^{(\alpha)}$.

On the other hand, let $\beta=\alpha+1$. For each $i$, we can choose a uniform cover $\mathcal{Q}_{i}$ such that $\mathcal{S}_{i} \mid U \in(\mu \mid U)_{0}^{(\alpha)}$ for all $U \in \mathcal{Q}_{l}$. Let $\mathcal{Q}=\left\{U_{j}\right\}_{j}$ be a uniform cover such that $\mathcal{Q}_{<}<\mathscr{Q}_{1} \wedge \cdots \wedge \mathcal{Q}_{n}$. We contend that for each $j, \mathscr{V} \mid U_{j} \in\left(\mu \mid U_{j}\right)_{0}^{(\alpha)}$. To prove this, note that $U_{j} \cap\left(X \sim \mathrm{cl}_{X} W_{i}\right) \subset$ $U_{j} \sim \operatorname{cl}_{U(j)}\left(W_{i} \cap U_{j}\right)$, and therefore the binary cover $\left\{V_{i} \cap U_{j}\right.$, $\left.U_{j} \sim \operatorname{cl}_{U(j)}\left(W_{i} \cap U_{j}\right)\right\}$ belongs by (ii) to $\left(\mu \mid U_{j}\right)_{0}^{(\alpha)}\left(\right.$ since $\operatorname{cl}_{U(j)}\left(W_{l} \cap U_{j}\right) \subset$ $\left.\mathrm{cl}_{X} W_{i} \cap U_{j} \subset V_{l} \cap U_{J}\right)$. By the induction hypothesis, $\mathcal{V} \mid U_{j} \in\left(\mu \mid U_{j}\right)_{0}^{(\alpha)}$, as desired. But then $\mathcal{V} \in \mu_{0}^{(\alpha+1)}$. This completes the induction. Hence $P(\alpha)$ holds for all ordinals $\alpha$.

To show that (ii) implies (iii), let $\mathcal{V}=\left\{V_{1}, \ldots, V_{n}\right\}$ be a finite open cover of $X$. Since by (ii) each binary open cover of $X$ belongs to the fine uniformity of $X$, the space $X$ is normal and thus we can find an open refinement $\left\{W_{1}, \ldots, W_{n}\right\}$ of $\mathcal{V}$ such that $\mathrm{cl}_{X} W_{i} \subset V_{i}$. Condition (ii) guarantees that each of the covers $\left\{V_{i}, X \sim \mathrm{cl}_{X} W_{i}\right\}$ belongs to $\mu_{0}^{(\alpha)}$. It follows from $P(\alpha)$ that $\mathscr{V} \in \mu_{0}^{(\alpha)}$.

LeMMA 3.2. Let $\rho X$ be a metric space of finitary rank $\alpha<\omega_{1}$. Then there is a compact $K \subset X$ such that each point of $X \sim K$ has a neighbourhood of finitary rank $<\alpha$.

Proof. Let $A$ be the set of all points $x \in X$ that admit no neighbourhood of finitary rank $<\alpha$. We will show that $A$ is compact. Since $A$ is a 
closed subspace of $X$ and $\rho X$ is ranked, $A$ is complete. If $A$ is not compact, then it is not totally bounded. Accordingly, we can choose an infinite subset $\left\{x_{m}: m \in N\right\}$ of $A$ that is uniformly discrete relative to some $\varepsilon>0$. We can assume $\alpha$ is a limit ordinal since the case of a successor ordinal is essentially similar, but simpler. Let $\{\beta(n): n \in N\}$ be an enumeration of the set of ordinals smaller than $\alpha$. Since $x_{m}$ does not have a neighbourhood of finitary rank $<\alpha$, the closed sphere $S_{m}=$ $\bar{S}_{\rho}\left(x_{m}, \varepsilon / 3\right)$ contains two closed disjoint sets $E_{m}$ and $F_{m}$ for which there is no cover $\mathscr{V} \in\left(\rho \mid S_{m}\right)_{0}^{(\beta(m))}$ with $\operatorname{St}\left(E_{m}, \mathcal{V}\right) \subset S_{m} \sim F_{m}$. (Here we use Lemma 3.1!) Let $E=\bigcup\left\{E_{m}: m \in N\right\}$ and $F=\bigcup\left\{F_{m}: m \in N\right\}$. Then $E$ and $F$ are closed and disjoint subsets of $X$; consequently, there is a $\mathcal{V} \in \mu_{0}^{(\alpha)}$ such that $\operatorname{St}(E, \mathcal{T}) \subset X \sim F$. Since $\alpha$ is a limit ordinal there is an $n \in N$ with $\mathscr{V} \in \mu_{0}^{(\beta(n))}$ (since $\mu_{0}^{(\alpha)}=\bigcup\left\{\mu_{0}^{(\beta(k))}: k \in N\right\}$ ). But $\operatorname{St}\left(E_{n}, \mathcal{V} \mid S_{n}\right) \subset S_{n} \sim F_{n}$ and $\mathcal{V} \mid S_{n} \in\left(\mu \mid S_{n}\right)^{(\beta(n))}$, which is a contradiction. Thus, $A$ is compact. It is obvious that $A$ satisfies the condition stated for $K$ in 3.2 .

COROLlaRY 3.3. Let $\rho X$ be a metric space of countable finitary rank. Then $X$ is $C$-scattered.

Proof. If rank $\rho X=0$, then $X$ is $C$-scattered by Nagata's theorem (Theorem 3 in [5]). Now proceed by induction on the finitary rank of $\rho X$ using 3.2.

Thus, it follows from a result of R. Telgársky ([8], Theorem 1.7) that a metric space of countable finitary rank is $F_{\sigma^{-}}$and $G_{\delta^{-}}$-absolute.

LEMMA 3.4. Let $\rho X$ be a metric space such that each point of $X$ admits a neighbourhood of finitary rank $<\alpha<\omega_{1}$, and suppose the finitary rank of $\rho X$ is $\leq \alpha$. Then there is an ordinal $\beta<\alpha$ and an $\varepsilon>0$ such that the finitary rank of each closed sphere of radius $\varepsilon$ is at most $\beta$.

Proof. Let $\{\beta(n): n \in N\}$ be an enumeration of the set of ordinals smaller than $\alpha$. The result follows from the observation that there is no infinite subset $E=\left\{x_{n}: n \in N\right\}$ of $X$ such that the finitary rank of $\bar{S}_{\rho}\left(x_{n}, 1 / n\right)$ is greater than $\beta(n)$. (Recall that $\rho X$ is complete and note that either $E$ contains a convergent sequence or a uniformly discrete sequence. The first case contradicts 3.4, while the second has been handled in the proof of 3.2.)

THeOrem 3.5. Let $\alpha<\omega_{1}$. A metric space $\rho X$ is of finitary rank $\alpha$ if, and only if, there is a compact subset $K \subset X$ satisfying the following 
condition: given any $\varepsilon>0$, there exist $\beta<\alpha$ and $\eta>0$ such that for each $x \in X \sim S_{\rho}(K, \varepsilon)$, the finitary rank of $\bar{S}_{\rho}(x, \eta)$ is at most $\beta$.

Proof. Theorem 3.5 follows directly from 3.2 and 3.4.

The following corollary can be proved by transfinite induction on the rank.

COROllary 3.6. A metric space is of finitary rank $<\omega_{1}$ if and only if the space is of rank $<\omega_{1}$.

We conclude this section by considering finite products of metric spaces. Telgársky proved in [7] that if $X$ and $Y$ are scattered spaces, then the Cantor-Bendixson rank of their product is

$$
\xi(X \times Y)=\sup \{\alpha \oplus \beta+1: \alpha<\xi(X), \beta<\xi(Y)\},
$$

where $\xi(X)$ and $\xi(Y)$ are the Cantor-Bendixson ranks of $X$ and $Y$, respectively. The following theorem shows that the Hessenberg sum can be used in connection with countable ranks in our sense. I do not know if the result can be extended to arbitrary ranks.

THEOREM 3.7. Let $\rho X$ and $\sigma Y$ be metric spaces with rank $\rho X=\alpha<\omega_{1}$ and rank $\sigma Y=\beta<\omega_{1}$. Then the product space $\rho X \times \sigma Y$ has rank $\leq \alpha \oplus \beta$ +1 .

Proof. We will proceed by induction on $\alpha \oplus \beta$. The case $\alpha \oplus \beta=0$ can be proved as the corresponding case $\alpha \oplus \beta=\gamma$ below. Thus, suppose the statement of 3.7 holds whenever $0 \leq \alpha \oplus \beta<\gamma$. Let $\alpha \oplus \beta=\gamma$. By 3.5 there exist compact sets $K_{1} \subset X, K_{2} \subset Y$ such that for each $\varepsilon>0$ we can find $\nu<\alpha, \tau<\beta, \eta_{1}>0, \eta_{2}>0$ with the following property: if $x \in X_{\varepsilon}=X \sim S_{\rho}\left(K_{1}, \varepsilon\right)$ (resp. $y \in Y_{\varepsilon}=Y \sim S_{\sigma}\left(K_{2}, \varepsilon\right)$ ) then $\bar{S}_{\rho}\left(x, \eta_{1}\right)$ is of rank $\leq \nu$ (resp. $\bar{S}_{\sigma}\left(y, \eta_{2}\right)$ is of rank $\left.\leq \tau\right)$. Put $\eta=\min \left\{\eta_{1}, \eta_{2}\right\}$. Now

$$
\begin{aligned}
(X \times Y) & \sim S_{\rho x \sigma}\left(K_{1} \times K_{2}, \varepsilon\right) \\
& =(X \times Y) \sim\left(S_{\rho}\left(K_{1}, \varepsilon\right) \times S_{\sigma}\left(K_{2}, \varepsilon\right)\right) \\
& =\left(X \times Y_{\varepsilon}\right) \cup\left(X_{\varepsilon} \times Y\right) .
\end{aligned}
$$

Choose a point $(x, y) \in\left(X \times Y_{\varepsilon}\right) \cup\left(X_{\varepsilon} \times Y\right)$. For example, let $x \in X_{\varepsilon}$ and $y \in Y$. Then $\operatorname{rank} \bar{S}_{\rho}(x, \eta) \leq \nu$. As $\oplus$ is strictly increasing, we have $\nu \oplus \beta<\gamma$ and thus by the induction hypothesis the rank of $\bar{S}_{\rho}(x, \eta)$ $\times \bar{S}_{\rho}(y, \eta)$ is $\leq \nu \oplus \beta+1=(\nu+1) \oplus \beta \leq \alpha \oplus \beta$. Similarly if $x \in X$ 
and $y \in Y_{\varepsilon}$, then the rank of $\bar{S}_{\rho}(x, \eta) \times \bar{S}_{\sigma}(y, \eta)$ is $\leq \alpha \oplus \tau+1=\alpha \oplus$ $(\tau+1) \leq \alpha \oplus \beta$. Therefore, the set $(X \times Y) \sim S_{\rho x \sigma}\left(K_{1} \times K_{2}, \varepsilon\right)$ admits a uniform cover by spheres of rank $\leq \alpha \oplus \beta$. As a consequence, 3.5 applies to show that the rank of $\rho X \times \sigma Y$ is $\leq \alpha \oplus \beta+1$.

CoRollaRY 3.8. Let $\rho_{1} X_{1}, \ldots, \rho_{n} X_{n}$ be metric spaces with rank $\rho_{i} X_{i}=$ $\alpha_{l}<\omega_{1}$. Then rank $\Pi\left\{\rho_{i} X_{l}: 1 \leq i \leq n\right\} \leq \alpha_{1} \oplus \cdots \oplus \alpha_{n}+(n-1)$.

4. Separable complete metric spaces. We shall prove the main result stating that the important class of $\sigma$-compact, complete metric spaces coincides with the class of separable metric spaces of countable Ginsburg-Isbell rank. The following result is due to Telgársky ([9], 12.2.4):

THEOREM 4.1. A C-scattered metric space admits a $\sigma$-discrete cover by compact sets.

COROllary 4.2. A separable, complete metric space is C-scattered if and only if it is $\sigma$-compact.

In fact, necessity in 4.2 follows immediately from 4.1 and sufficiency is a direct consequence of Baire's category theorem.

LEMMA 4.3. Let $P$ be a countable partially ordered set and assume $S$ is a subset of $P$ such that each descending chain in $P \sim S$ is finite. Let $\left\{U_{p}\right.$ : $p \in P\}$ be a uniform cover of a uniform space $\mu X$ such that for each $p \in P$, the restriction of $\left\{U_{q}: q\right.$ a successor of $\left.p\right\}$ to $U_{p}$ is a uniform cover of $U_{p}$. Then there is an $\alpha<\omega_{1}$ such that $\mathscr{V}_{S}=\left\{U_{p}: p \in S\right\} \in \mu^{(\alpha)}$.

Proof. A corresponding statement was proved in [3], VII.8. The same method of proof applies here.

LEMMA 4.4. Let $\rho X$ be a complete separable metric space and let $\mathfrak{T}$ be an open cover of $X$. Then there is an $\alpha<\omega_{1}$ such that $\mathscr{V} \in \mu^{(\alpha)}$.

Proof. For each $n$ let $\mathcal{Q}_{n}$ be the cover of $X$ consisting of all spheres with radius $2^{-n}$. Since $X$ is separable, $\mathcal{U}_{n}$ has a uniform countable shrinking $\left\{U_{n, \alpha}: \alpha \in A_{n}\right\}$. Then proceed as in [3], VII.9., and use Lemma 4.3.

COROLlaRY 4.5. If $\rho X$ is a complete separable metric space, the Ginsburg-Isbell rank of $\rho X$ is $\leq \omega_{1}$. 
The irrationals $J$ form a $G_{\delta}$-subset of the reals and thus by a theorem due to P. Alexandroff, $J$ admits a complete metric $\rho$. It follows from 4.5 that the Ginsburg-Isbell rank of $\rho J$ is at most $\omega_{1}$. On the other hand, if that rank were a countable ordinal, then by $2.3 \operatorname{rank} \rho J<\omega_{1}$, and by $3.3 J$ would be $C$-scattered. Hence, the Ginsburg-Isbell rank of $\rho J$ is $\omega_{1}$. The same argument shows that the Ginsburg-Isbell rank of an infinite-dimensional separable Banach space is $\omega_{1}$.

THEOREM 4.6. Let $\rho X$ be a separable complete metric space. Then the following statements are equivalent:

(i) $X$ is $\sigma$-compact;

(ii) the Ginsburg-Isbell rank of $\rho X$ is countable.

Proof. By 3.3 and 4.2 it is enough to show that (i) implies (ii). To this end, let $X$ be $\sigma$-compact. By 4.2, $X$ is $C$-scattered. As $X$ is $C$-scattered, we can choose a point $x_{0} \in X$ and find an open neighbourhood $U_{0}$ of $x_{0}$ such that $\mathrm{cl}_{X} U_{0}$ is compact. Initially, let $F_{0}=X$. Suppose the sequence $\left\langle U_{\alpha}, F_{\alpha}\right\rangle_{\alpha<\beta}$ has been defined and $\beta \geq 1$. Let $F_{\beta}=X \sim \cup\left\{U_{\alpha}: \alpha<\beta\right\}$. Then $F_{\beta}$ is a closed subset of $X$, whence there is a point $x_{\beta} \in F_{\beta}$ and an open neighbourhood $U_{\beta}$ of $x_{\beta}$ in $X$ such that $\mathrm{cl}_{X} U_{\beta} \cap F_{\beta}$ is compact. Clearly there is an ordinal $\beta$ such that $X=\cup\left\{U_{\alpha}: \alpha<\beta\right\}$. We call $\left\langle U_{\alpha}, F_{\alpha}\right\rangle_{\alpha<\beta}$ an exhaustion of length $\beta$. If $X$ has an exhaustion of length 1, then $X$ is compact. Now we shall proceed by induction on the length of exhaustion. Thus, assume that if a complete separable metric space has an exhaustion of length $\beta$, where $1 \leq \beta<\gamma<\omega_{1}$, then the Ginsburg-Isbell rank of the space is countable. Let $X$ have an exhaustion $\left\langle U_{\alpha}, F_{\alpha}\right\rangle_{\alpha<\gamma}$ of length $\gamma$. We have two cases to consider. First, let $\gamma=\beta+1$. Then $X=\bigcup\left\{U_{\alpha}: \alpha<\beta\right\} \cup U_{\beta}$. Therefore $F_{\beta}$ is a closed subspace of $\mathrm{cl}_{X} U_{\beta}$ and thus $F_{\beta}$ is compact. Let $Y_{n}=X \sim S_{\rho}\left(F_{\beta}, 1 / n\right)$. Then $Y_{n}$ is complete and separable. Moreover, clearly $Y_{n}$ has an exhaustion of length $\leq \beta<\gamma$, whence by the induction hypothesis the Ginsburg-Isbell rank of $Y_{n}$ is some countable ordinal $\tau_{n}$. Let $\tau=\sup \left\{\tau_{n}: n \in N\right\}<\omega_{1}$ and let $\mathscr{V}$ be an open cover of $X$. As $F_{\beta}$ is compact, we can find a finite subfamily $\mathcal{V}^{\prime} \subset \mathcal{V}$ and an $n$ such that $S_{\rho}\left(F_{\beta}, 4 / n\right) \subset \cup\left(\mathcal{V}^{\prime}\right)$ and $\mathcal{V}^{\prime}$ forms a uniform cover of $S_{\rho}\left(F_{\beta}, 2 / n\right)$. Now $\mathscr{V} \mid Y_{n} \in\left(\rho \mid Y_{n}\right)^{(\tau(n))}$. On the other hand, note that $\left\{Y_{n}, S_{\rho}\left(F_{\beta}, 2 / n\right)\right\}$ is a uniform cover of $X$. It follows that $\mathcal{V} \in \rho^{(\tau(n)+1)} \subset$ $\rho^{(\tau+1)}$. Therefore, every open cover of $X$ belongs to $\rho^{(\tau+1)}$. Thus, suppose $\gamma$ is a limit ordinal. For each $\beta<\gamma$, the set $\operatorname{cl}_{X} U_{\beta}$ is of some countable Ginsburg-Isbell rank $\tau_{n}$. (To see this, use the above argument with $F_{\beta}$ replaced by $\mathrm{cl}_{X} U_{\beta} \cap F_{\beta}$.) As $\rho X$ is complete and separable, 4.4 implies 
there is a $\nu<\omega_{1}$ such that $\left\{\operatorname{cl}_{X} U_{\beta}: \beta<\gamma\right\} \in \rho^{(\nu)}$. Let $\mathcal{V}$ be an open cover of $X$. Then for each $\beta<\gamma$, we have $\mathcal{V} \mid \mathrm{cl}_{X} U_{\beta} \in\left(\rho \mid \mathrm{cl}_{X} U_{\beta}\right)^{(\tau(n))}$. Thus $\mathcal{T}$ belongs to $\rho^{(\tau)}$, where $\tau=\max \left\{\sup \left\{\tau_{n}: n \in N\right\}, \nu\right\}+1<\omega_{1}$. This completes our inductive proof, since every $C$-scattered separable metric space has an exhaustion of countable length.

Theorem 4.6 together with 3.5 enables us to give an analysis of the structure of $\sigma$-compact complete metric spaces. Let $\rho X$ be such a space. By $4.6 \rho X$ is of countable rank and thus by 3.5 there is a compact $K \subset X$ such that each point $x$ in $X \sim K$ admits a neighbourhood $U_{X}$ of lower rank. The same applies to each $U_{X}$. Hence, we obtain the following tree-like presentation of $X$.

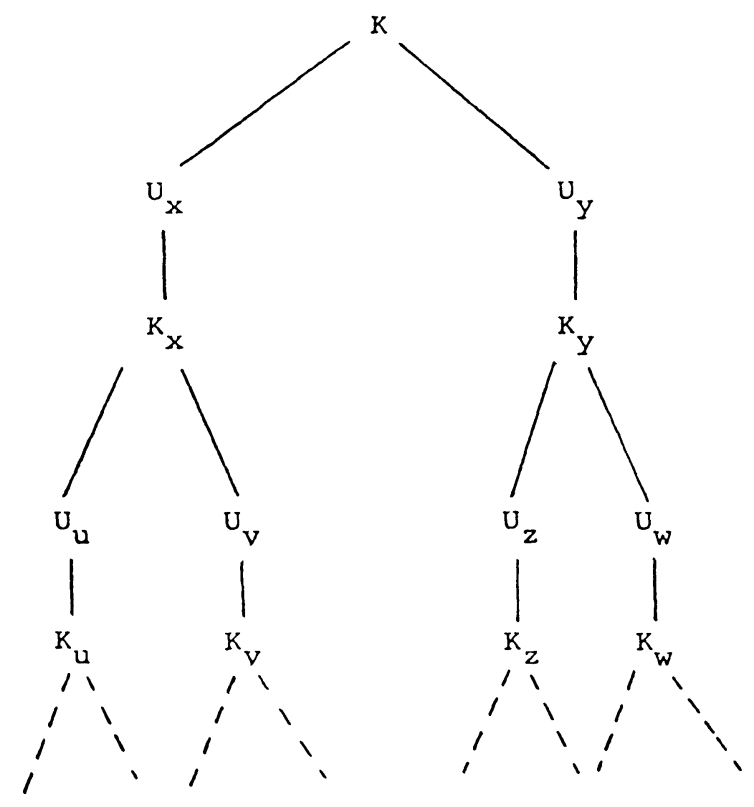

Suppose $\cdots \supset U_{x(n)} \supset U_{x(n+1)} \supset U_{x(n+2)} \supset \cdots$ is a part of a branch of this tree and let $\cdots>\operatorname{rank} U_{x(n)}>\operatorname{rank} U_{x(n+1)}>\operatorname{rank} U_{x(n+2)}>\cdots$. As there are no infinite decreasing sequences of ordinals, the above sequence stops at some $U_{x(m)}$. But $U_{x(m)}$ is compact, for otherwise we can find a point $x_{m+1} \in U_{x(m)}$ with a neighbourhood $U_{x(m+1)}$ such that either $U_{x(m+1)}$ $=\left\{x_{m+1}\right\}$ or rank $U_{x(m+1)}<\operatorname{rank} U_{x(m)}$. Hence the branches of the above tree are finite and their endpoints are compact sets. Since these trees give an analysis of $\sigma$-compact complete metric spaces, it seems worth while to study their structure. (There have been studies on tree-structures in 
connection with Ginsburg-Isbell derivatives; see [6].) However, that study is outside the scope of this paper.

REMARK. It seems that many of our results can be extended to linearly uniformizable spaces (i.e. $\omega_{\alpha}$-metrizable spaces).

Acknowledgements. I am grateful to Heikki Junnila for advice and help during the preparation of this paper. R. Telgársky pointed out the relevance of the Hessenberg sum for the product Theorem 3.7. Professor Nagata kindly informed me of his early work on equinormal uniform spaces.

\section{REFERENCES}

[1] S. Ginsburg and J. R. Isbell, Some operators on uniform spaces, Trans. Amer. Math. Soc., 93 (1959), 145-168.

[2] A. Hohti, On uniform paracompactness, Ann. Acad. Scient. Fenn., Series A, I. Mathematica Dissertationes 36 (1981).

[3] J. R. Isbell, Uniform spaces, Mathematical Surveys No. 12, American Mathematical Society, Providence, Rhode Island, 1964.

[4] K. Kuratowski and A. Mostowski, Set Theory, North-Holland Publishing Company, Amsterdam, 1976.

[5] J. Nagata, On the uniform topology of bicompactifications, Journal of the Institute of Polytechnics, Osaka City University, Vol. 1, No. 1. Series A, 1950.

[6] J. Pelant and M. D. Rice, Remarks on e-locally fine spaces, Seminar Uniform Spaces 1976-1977, ČSAV, Prague, pp. 51-62.

[7] R. Telgársky, Derivatives of cartesian product and dispersed spaces, Coll. Math. 29 : 1, (1968), 59-66.

[8] __, C-scattered and paracompact spaces, Fund. Math., 73 (1972), 59-74.

[9] __ Spaces defined by topological games, Fund. Math., $78: 3$ (1975), 193-223.

Received January 20, 1982 and in revised form April 5, 1983.

UNIVERSITY OF HELSINKI

SF-00100 HELSINKI 10

FINLAND 


\section{PACIFIC JOURNAL OF MATHEMATICS}

EDITORS

Donald BabBITT (Managing Editor)

University of California

Los Angeles, CA 90024

Hugo Rossi

University of Utah

Salt Lake City, UT 84112

C. C. Moore and Arthur Ogus

University of California

Berkeley, CA 94720
J. DugunduI

Department of Mathematics

University of Southern California

Los Angeles, CA 90089-1113

R. FINN and H. SAMELSON

Stanford University

Stanford, CA 94305

\section{ASSOCIATE EDITORS}
R. ARENS
E. F. BECKENBACH
B. H. NeumanN
F. WolF
K. YosHIDA (1906-1982)

\section{SUPPORTING INSTITUTIONS}

UNIVERSITY OF ARIZONA

UNIVERSITY OF BRITISH COLUMBIA

CALIFORNIA INSTITUTE OF TECHNOLOGY

UNIVERSITY OF CALIFORNIA

MONTANA STATE UNIVERSITY

UNIVERSITY OF NEVADA. RENO

NEW MEXICO STATE UNIVERSITY

OREGON STATE UNIVERSITY
UNIVERSITY OF OREGON

UNIVERSITY OF SOUTHERN CALIFORNIA

STANFORD UNIVERSITY

UNIVERSITY OF HAWAII

UNIVERSITY OF TOKYO

UNIVERSITY OF UTAH

WASHINGTON STATE UNIVERSITY

UNIVERSITY OF WASHINGTON 


\section{Pacific Journal of Mathematics}

\section{Vol. 111, No. $1 \quad$ November, 1984}

Harald Brandenburg and Adam Stefan Mysior, For every Hausdorff

space $Y$ there exists a nontrivial Moore space on which all continuous

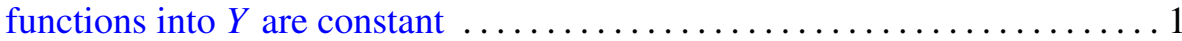

Henry Dappa, A Marcinkiewicz criterion for $L^{p}$-multipliers $\ldots \ldots \ldots \ldots \ldots 9$

P. H. Doyle, III and John Gilbert Hocking, Bijectively related spaces. I.

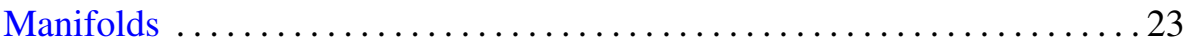

Joel Hass, Complete area minimizing minimal surfaces which are not totally

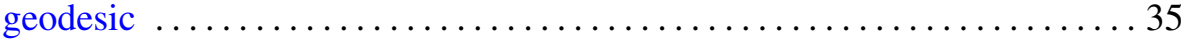

Aarno Hohti, On Ginsburg-Isbell derivatives and ranks of metric spaces .... 39

Richard Howard Hudson, Diophantine determinations of $3^{(p-1) / 8}$ and $5^{(p-1) / 4}$

A. F. Izé and A. Ventura, Asymptotic behavior of a perturbed neutral functional-differential equation related to the solution of the unperturbed

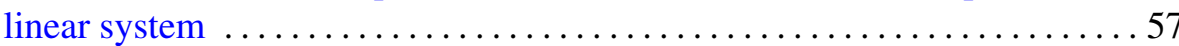

Palle E. T. Jorgensen, Spectral representations of unbounded nonlinear

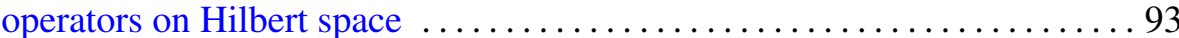

Darrell Conley Kent and Gary Douglas Richardson, Cauchy spaces with

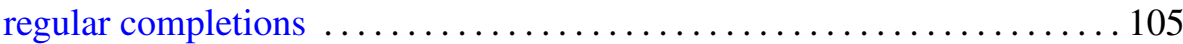

Mark Mahowald, An addendum to: "bo-resolutions" ................ 117

Stuart Wayne Margolis and Jean-Eric Pin, Minimal noncommutative varieties and power varieties

Carla Massaza and Alfio Ragusa, Some conditions on the homology groups of the Koszul complex

Vicente Miquel Molina, Some examples of Riemannian almost-product manifolds

Roderic Murufas, Inverse spectral problems for certain differential operators

Ulrich Oertel, Closed incompressible surfaces in complements of star links

Katsuro Sakai, A characterization of local equiconnectedness

William Victor Smith and Don Harrell Tucker, Weak integral convergence theorems and operator measures 